\title{
Numeralität als soziale Praxis - forschungstheoretische Einordnung und empirische Zugänge
}

\author{
Antje Pabst • Wiebke Curdt • Melanie Benz-Gydat • \\ Silke Schreiber-Barsch • Christine Zeuner
}

Eingegangen: 4. Juli 2019 / Angenommen: 16. September 2019 / Online publiziert: 18. Oktober 2019 (C) Der/die Autor(en) 2019

Zusammenfassung Numeralität als Teil von Grundbildung wurde im deutschen Sprachraum bisher sowohl in der Wissenschafts- als auch in der Praxisdiskussion der Erwachsenenbildung nur peripher berücksichtigt. Ausgehend von diesem Befund, führt der Beitrag die einzelnen forschungstheoretischen und konzeptionellen Linien des Diskursraumes in einer Systematik zusammen. Zudem argumentiert der Beitrag für eine Erweiterung des Diskurses um den Ansatz einer subjektorientierten Grundbildung, der auf dem Konzept von Numeralität als soziale Praxis aufbaut. Erste Befunde aus zwei Forschungsprojekten zur Verschränkung von Numeralität mit Alter bzw. Behinderung können subjektive Begründungs- und Bedeutungszusammenhänge sowie die soziale Einbettung von Numeralität aufdecken.

Schlüsselwörter Numeralität als soziale Praxis · Alltagsmathematik · Grundbildung · Partizipation · Ältere · Behinderung · Qualitative Forschung

Dr. A. Pabst · Dr. M. Benz-Gydat · Prof. Dr. C. Zeuner

Helmut-Schmidt-Universität/Universität der Bundeswehr Hamburg, Hamburg, Deutschland

Dr. A. Pabst

E-Mail: antje.pabst@hsu-hh.de

Dr. M. Benz-Gydat

E-Mail: benz-gydat@hsu-hh.de

Prof. Dr. C. Zeuner

E-Mail: zeuner@hsu-hh.de

Dr. W. Curdt · JProf. Dr. S. Schreiber-Barsch ( $\square)$

Universität Hamburg, Hamburg, Deutschland

E-Mail: silke.schreiber-barsch@uni-hamburg.de

Dr. W. Curdt

E-Mail: wiebke.curdt@uni-hamburg.de 


\section{Numeracy as social practice - current research and empirical approaches}

Abstract So far, numeracy as part of basic education has only been considered peripherally in the German-speaking countries, regarding the scientific as well as the practical approaches of adult education. In this article, theoretical and conceptual lines of the discourse are linked systematically. In addition, it is argued for an extension of the discourse by including the concept of subject-oriented basic education, which is based on the concept of numeracy as social practice. Initial findings from two research projects, one looking at the use of numeracy by older people and the other one looking at people with disabilities, reveal subjective contexts of justification and meaning as well as the social embeddedness of numeracy.

Keywords Numeracy as social practice $\cdot$ Everyday mathematics $\cdot$ Basic education · Participation · People of older age · Disability - Qualitative research

\section{Einleitung}

Seit mehr als fünfzehn Jahren stehen Alphabetisierung und Grundbildung Erwachsener auf der bildungspolitischen Agenda der Bundesrepublik Deutschland. So bildete sich ein Bereich der Erwachsenenbildung, der seit den 1970er Jahren ein Nischendasein führte, zu einem breit diskutierten Themenfeld der Praxis sowie der erziehungs- und bildungswissenschaftlichen Theorie und Forschung heraus. Im Rahmen der Grundbildung werden die Vielfalt und Komplexität grundlegender Fähigkeiten und Fertigkeiten Erwachsener diskutiert, die gesellschaftlich erwartet bzw. individuell gebraucht werden. Ihnen kommt eine besondere Bedeutung für die politische und gesellschaftliche Partizipation und die Selbst- und Mitbestimmung der Individuen $\mathrm{zu}$.

Numeralität als Teil von Grundbildung blieb in der wissenschaftlichen Diskussion im deutschen Sprachraum und in der Praxis der Erwachsenenbildung bislang weitgehend unbeachtet (z. B. Kittel 2016). Unser Beitrag zielt auf eine grundlegende Erweiterung sowohl der Debatte um Numeralität als auch der Forschungsperspektiven auf diesen Gegenstand. Dabei legen wir unseren Ausführungen den forschungstheoretischen Ansatz Numeralität als soziale Praxis (NSP) (vgl. z. B. Yasukawa et al. 2018b) in Anlehnung an die Tradition der New Literacy Studies (Street 1984; The New London Group 1996) zugrunde.

Wird Numeralität als soziale Praxis verstanden, so wird der Blick auf individuelle Anwendungen und Bedeutungszuschreibungen sowie gesellschaftliche Anforderungs- und Machtstrukturen gelenkt. Dies kann zum einen zeigen, welche gesellschaftlichen und kulturellen Vorstellungen von Numeralität das Leben und Handeln der Menschen prägen. Zum anderen wird erkennbar, auf welche Art und Weise Menschen sich Numeralität subjektiv zu eigen machen, praktizieren und nutzen. Es geht um ihre Verwendung innerhalb gesellschaftlicher und sozialer Kontexte, unabhängig von einem erwarteten formalisierten Beherrschungsgrad von Fähigkeiten und Fertig- 
keiten, der bspw. grundlegend für Verfahren einer quantitativen Kompetenzmessung ist.

Einem solchen Verständnis folgend, legen wir im vorliegenden Beitrag nach einer einführenden Systematisierung (Abschn. 2) unsere forschungstheoretischen Auslegungen des Konzepts Numeralität als soziale Praxis dar und präsentieren Zwischenergebnisse aus zwei aktuellen Forschungsprojekten, die Numeralität zum einen in Bezug auf Alter und in Bezug auf Behinderung/Disability zum anderen untersuchen (Abschn. 3). Abschließend werden Impulse für die Debatte um Grundbildung benannt (Abschn. 4).

\section{Numeralität als Begriff und Konzept}

Der Begriff „Numeralität“ als Begriff findet sich seit einigen Jahren als Gegenstand in der internationalen Diskussion und Forschung. Einige Studien entstanden bereits in den 1980er Jahren, wie bspw. der „Cockcroft Report“ (1982), der insbesondere eine statistische Kompetenz hervorhebt, damit Menschen selbsttätig beurteilen können, wo Meinungen beeinflusst oder getäuscht werden. Jean Lave (1988) untersuchte alltagsbezogenes Lernen mithilfe numeraler Praktiken. Anfang der 2000er Jahre diagnostizierten Coben et al. (2003) eine begriffliche Diffusität (ebd., S. 393), zehn Jahre später systematisierten Geiger et al. (2015) die unterschiedlichen theoretischen Perspektiven auf und Ansätze von Numeralität.

Der Beitrag folgt dem Verständnis von Numeralität als einem weiten Begriff (vgl. Geiger et al. 2015), in dem Konzepte standardisierter schulischer Mathematik ebenso wie die Perspektive auf alltägliche numerale Routinen enthalten sind. Leitend ist zudem das Konzept Numeralität als soziale Praxis (NSP). Dieser Ansatz untersucht, was Menschen tagtäglich bzw. im Rahmen unterschiedlicher gesellschaftlicher Bereiche und in verschiedenen sozialen Interaktionen mit Numeralität machen und geht damit über das Ausführen einer individuellen mathematischen Leistung (im Sinne sog. Schulmathematik) hinaus. Jene wird oft vom sozialen Kontext isoliert betrachtet und zielt überwiegend auf die Anwendung universeller mathematischer Operationen ab, die standardisiert und vergleichbar sind (Yasukawa et al. 2018a, S. 13). Demgegenüber konzentriert sich NSP auf die Praxis, die kulturell, historisch und auch politisch gestaltet ist (ebd.), und in der sichtbare und unsichtbare numerale Artefakte und Handlungen eingebettet sind. In den Blick geraten Anwendungsbereiche und damit subjektiv begründetes Alltagshandeln der Menschen unter Bezugnahme auf mathematisches Wissen, Fähigkeiten und Fertigkeiten. Ein solch subjektorientierter Blick auf Numeralität fragt nach den individuellen Bedeutungszuschreibungen sowie den Denk-, Reflexions- und Handlungsmustern im Alltag.

Das Konzept von NSP gründet laut Yasukawa et al. (2018a, S. 5ff.) auf vier theoretischen Ursprüngen und Forschungslinien:

- Erstens auf Studien zur situativen und praxisbezogenen Sichtweise auf numerale Aktivitäten. Zentral ist der Begriff alltäglicher, routinierter Praxis, d.h., ,was Menschen in täglichen, wöchentlichen und gewöhnlichen Aktivitätszyklen tun“ (Lave 1988, S. 15). 
- Zweitens sind es Ansätze, die sich auf Wygotskys (1986) kulturhistorische Tätigkeitstheorie beziehen. In diesem Zusammenhang betonen jüngere Studien die Unsichtbarkeit von Mathematik und fragen nach Lernprozessen im Rahmen von technischen und technologischen Veränderungen.

- Drittens ist der Einfluss der Ethnomathematik zu nennen (z. B. D’Ambrosio 1985). Sie berücksichtigt interkulturelle Aspekte bei der didaktischen und curricularen Gestaltung von Mathematik, damit für alle Lernenden die Chance auf Emanzipation und Mündigkeit besteht.

- Viertens sind schließlich die Studien zu Literalität als soziale Praxis (LSP) zu nennen, entstanden aus der Arbeit des Anthropologen Street (1984). Literalität bezieht sich dabei auf jede Form der sozialen Kommunikation oder Praxis, die einen semiotischen Code erfordert. Sowohl LSP als auch NSP integrieren Aspekte wie die soziale Einbettung und die subjektiven und gemeinschaftlichen Aneignungs- und Nutzungsprozesse.

Im deutschsprachigen Diskurs spielen Numeralität bzw. Alltagsmathematik Erwachsener bislang nur eine untergeordnete Rolle. Die Forschung zu mathematischen/ numeralen Kompetenzen Erwachsener erfolgt zudem überwiegend empirisch quantitativ, wie Tab. 1 verdeutlicht. Studien, die auf numerale bzw. alltagsmathematische Fähigkeiten und Fertigkeiten abzielen, und Curricula oder auch Konzepte im Hinblick auf einen pädagogischen Praxistransfer erarbeiten, existieren erst wenige.

Dennoch hat das Thema Numeralität im Rahmen internationaler Vergleichsstudien zur Kompetenzmessung, wie der PIAAC-Studie (2011/2012), öffentliche Wahrnehmung erfahren. Mit der deutschen PIAAC-Studie (Rammstedt 2013) liegt seither mit den repräsentativ erhobenen, international vergleichbaren Daten auch zu alltagsmathematischen Kompetenzen Erwachsener eine grundlegende Erweiterung des Forschungsstands vor. Die Ergebnisse von PIAAC beziehen sich auf die mathematischen Kenntnisse von 16- bis 64-Jährigen. Die deutschen Ergebnisse zeigen, dass die mathematischen Kenntnisse von knapp $50 \%$ der Befragten den Kompetenzniveaus 1 bis 3 zugerechnet werden können (Zabal et al. 2013, S. 55).

In den angeführten quantitativen Forschungszugriffen wird die alltägliche Relevanz von numeralem bzw. alltagsmathematischem Verhalten berücksichtigt, aber auf standardisierte und vergleichbare Weise erhoben bzw. gemessen, so dass kognitive Dimensionen und ein international harmonisiertes Verständnis von Numeralität die Ausgangsbasis bilden. Mit der methodologischen Prämisse der weitgehend kontextund kulturunabhängigen Konstruktion der Erhebungsverfahren, die auf einem engen Kompetenzverständnis aufbauen (vgl. Yasukawa et al. 2018a, S. 4), kann ein subjektiver und individueller Charakter numeralen Verhaltens jedoch nicht berücksichtigt werden bzw. ist quantitativ nicht abbildbar.

Davon ausgehend erscheinen uns Schlussfolgerungen problematisch, die für die Grundbildungspraxis gezogen wurden und die v. a. auf quantitativen Befunden der Kompetenzmessung beruhen. Es folgt oft eine defizitorientierte Interpretation der Messergebnisse, die dazu führt, dass aus als niedrig klassifizierten mathematischen Grundkenntnissen ohne weitere empirische Evidenz auf Biografien der Subjekte rückgeschlossen wird. So heißt es bspw. im Rahmencurriculum des DVV einführend: 
Tab. 1 Numeralität: Empirische Studien und Konzepte zum pädagogischen Praxistransfer im deutschsprachigen Raum. (Eigene Darstellung)

\begin{tabular}{|c|c|c|c|}
\hline \multirow[t]{2}{*}{$\begin{array}{l}\text { Forschungs- } \\
\text { typus }\end{array}$} & \multirow[t]{2}{*}{ Titel/Jahr } & \multirow{2}{*}{$\begin{array}{l}\text { GF: Sample/Erhebungszugriff } \\
\text { PT: Zielsetzung der Durchführung }\end{array}$} & \multirow{2}{*}{$\begin{array}{l}\text { GF: Forschungsparadigma/ } \\
\text { Forschungsgegenstand } \\
\text { PT: konzeptionelle Logik }\end{array}$} \\
\hline & & & \\
\hline \multirow[t]{4}{*}{$\begin{array}{l}\text { Grundlagen- } \\
\text { forschung } \\
\text { (GF) }\end{array}$} & $\begin{array}{l}\text { PIAAC } \\
\text { Deutschland } \\
(2011 / 2012) \\
\text { Rammstedt } \\
(2013)\end{array}$ & $\begin{array}{l}\text { 16- bis 65-Jährige } \\
\text { Repräsentative Zufallsstichpro- } \\
\text { be }(n=5379) \text {; ausgenommen: } \\
\text { Personen in institutionalisierten } \\
\text { Einrichtungen (Gefängnis, Heim- } \\
\text { einrichtung u. Ä.) }\end{array}$ & $\begin{array}{l}\text { Quantitativ } \\
\text { Kompetenzmessung: } \\
\text { u. a. alltagsmathematische } \\
\text { Kompetenzen }\end{array}$ \\
\hline & $\begin{array}{l}\text { CiLL-Studie I } \\
\text { und II } \\
\text { (2009; 2012) } \\
\text { u. a. Friebe } \\
\text { et al. (2014) }\end{array}$ & $\begin{array}{l}\text { Begleitstudie dt. PIAAC-Erhebung, } \\
\text { Erweiterung Stichprobe um Ältere: } \\
\text { 66- bis } 80-J a ̈ h r i g e ~(n=1339) \\
\text { Qualitative Fallstudien }(n=42)\end{array}$ & $\begin{array}{l}\text { Quantitativ \& qualitativ } \\
\text { Kompetenzmessung: } \\
\text { u. a. alltagsmathematische } \\
\text { Kompetenzen }\end{array}$ \\
\hline & $\begin{array}{l}\text { LEO-Level- } \\
\text { one-Studie II } \\
\text { (2018) } \\
\text { Grotlüschen } \\
\text { et al. (2019) }\end{array}$ & $\begin{array}{l}\text { 18- bis 64-Jährige } \\
\text { Repräsentative Zufallsstichprobe } \\
\text { ( } n=6681 ;+ \text { Zusatzstichprobe Per- } \\
\text { sonen im unteren Bildungsbereich, } \\
n=511)\end{array}$ & $\begin{array}{l}\text { Quantitativ } \\
\text { Kompetenzmessung: } \\
\text { u. a. Literalität und finanzbezo- } \\
\text { gene Praktiken }\end{array}$ \\
\hline & $\begin{array}{l}\text { Redmer und } \\
\text { Grotlüschen } \\
(2019)\end{array}$ & $\begin{array}{l}\text { Über-65-Jährige } \\
\text { Sekundäranalyse CiLL-Studie } \\
\text { (2012) \& PIAAC (2011/2012) }\end{array}$ & $\begin{array}{l}\text { Quantitativ } \\
\text { Kompetenznutzung: } \\
\text { Kompetenznutzungsvariablen } \\
\text { von finanzbezogenen Prakti- } \\
\text { ken }\end{array}$ \\
\hline $\begin{array}{l}\text { Im Über- } \\
\text { gang: } \\
\text { Grundlagen- } \\
\text { forschung } \\
\text { \& Praxis- } \\
\text { transfer }\end{array}$ & $\begin{array}{l}\text { Finanzielle } \\
\text { Grundbildung } \\
\text { Mania und } \\
\text { Tröster (2014, } \\
\text { 2015) }\end{array}$ & $\begin{array}{l}\text { Experteninterviews mit Schuld- } \\
\text { nerberater/inne/ } n(n=14) \& \\
\text { mit professionell Tätigen der } \\
\text { Erwachsenen-/Weiterbildung } \\
(n=10) \text {; Forschende Lernwerk- } \\
\text { stätten ( } n=18 \text { Ratsuchende aus } \\
\text { Schuldnerberatung) }\end{array}$ & $\begin{array}{l}\text { Qualitativ } \\
\text { Finanzielle Grundbildung als } \\
\text { Alltagskompetenz: } \\
\text { basale, lebenspraktische An- } \\
\text { forderungen alltäglichen Han- } \\
\text { delns und der Lebensführung } \\
\text { in geldlichen Angelegenheiten }\end{array}$ \\
\hline \multirow[t]{2}{*}{$\begin{array}{l}\text { Praxistrans- } \\
\text { ferkonzepte } \\
(\text { PT) }\end{array}$} & $\begin{array}{l}\text { Bausteine zur } \\
\text { Förderung } \\
\text { alltagsma- } \\
\text { thematischer } \\
\text { Kompetenz } \\
\text { Kaiser (2009) }\end{array}$ & $\begin{array}{l}\text { Instrumente zur erwachsenenpäd- } \\
\text { agogischen Kompensation alltags- } \\
\text { mathematischer Kompetenzen }\end{array}$ & $\begin{array}{l}\text { Konzeptionelle Logik } \\
\text { Unsichtbarkeit von Alltagsma- } \\
\text { thematik verhindert Wahrneh- } \\
\text { men eigener Fertigkeiten und } \\
\text { begründet erwachsenenpäd- } \\
\text { agogische Intervention }\end{array}$ \\
\hline & $\begin{array}{l}\text { Rahmencurri- } \\
\text { culum Rech- } \\
\text { nen } \\
\text { DVV (2017) }\end{array}$ & $\begin{array}{l}\text { Stufeneinteilung einer sog. lebens- } \\
\text { tauglichen Mathematik; Kompen- } \\
\text { sation des schulmathematischen } \\
\text { Zugangs }\end{array}$ & $\begin{array}{l}\text { Konzeptionelle Logik } \\
\text { Mathematische Lernbiogra- } \\
\text { phien als ,Scheiternsbiogra- } \\
\text { fien“ (S. 5) machen non-/ } \\
\text { formale erwachsenenpädago- } \\
\text { gische Intervention notwendig }\end{array}$ \\
\hline
\end{tabular}

Bei den Teilnehmern und Teilnehmerinnen von mathematischen Basiskursen muss man davon ausgehen, dass die mathematische Lernbiografie in einer Sackgasse gelandet bzw. eine Scheiternsbiografie ist. Dabei ist es tendenziell nicht so, dass die Teilnehmer/-innen nicht mathematisch denken. Es ist eher so, dass sie eine andere Mathematik als die konventionell übliche konstruiert 
haben. Diese Mathematik ist meist von fragmentarisch angehäuften Rechenregeln, Tricks und Kniffen geprägt. (Meyerhöfer 2017, S. 5)

Eine solche defizitorientierte Einschätzung widerspricht unserem forschungstheoretischen Zugang, wie wir nachfolgend verdeutlichen wollen. Mit Numeralität als soziale Praxis legen wir einen Begriff von Numeralität zugrunde, der jegliches mathematische Denken und Handeln berücksichtigt. Aus unserer Sicht ist anzuerkennen, dass Menschen ihre Fähigkeiten und Praktiken, sollten diese zu einem bestimmten Zeitpunkt Zielsetzung und Zweck nicht mehr erfüllen, selbsttätig und auf unterschiedlichen Wegen verändern oder auch erweitern.

\section{Erweiterung der empirischen Grundbildungsforschung - Einblicke in aktuelle Forschungsprojekte}

Vorgestellt werden Zwischenbefunde aus zwei aktuellen Forschungsprojekten, die Teil des Forschungsverbunds „Alltagsmathematik als Teil der Grundbildung Erwachsener" (2017-2020) sind. Ein Projekt fokussiert Grundbildung in Bezug auf die Kategorie Alter und beforscht „Numeralität als soziale Praxis im Wandel der Zeit“" (Zeuner; Pabst; Benz-Gydat). Ein weiteres widmet sich der Kategorie Behinderung bzw. dis/ability und untersucht „Numerale Praktiken von Menschen mit Lernschwierigkeiten“ (sog. intellektuellen Beeinträchtigungen; wir folgen der von People First, einer Selbstvertretungsorganisation, gesetzten Bezeichnung) (Schreiber-Barsch; Curdt; Gundlach; Eggersmann; Rückforth).

Beide Studien beziehen sich auf theoretische Überlegungen, die sich erstens auf die zwei Modelle (autonomes sowie ideologisches Modell) von Numeralität beziehen wie sie Street und Baker definiert haben (Street 1984; Baker und Street 1996). Im autonomen Modell wird Numeralität unabhängig vom sozialen Kontext neutrale Eigenschaften zugesprochen, die über Zeit und Raum universell sind und eher als verallgemeinerbar gelten denn als kulturell spezifisch. Das ideologische Modell betrachtet dagegen numerale Praktiken in kulturellen Kontexten, die immer von Machtverhältnissen durchdrungen sind und in denen Bedeutungen und Ressourcen konkurrieren (Baker und Street 1996, S. 79; Street et al. 2008).

Zweitens erweist sich die analytische Unterscheidung von literalen und numeralen Ereignissen und Praktiken als hilfreich (Heath 1982; Street 1984, S. 130f.).

Drittens ist die Differenzierung von alltagsweltlichen und dominanten Literalitäten (Street 1984) und Numeralitäten wesentlich. Barton und Hamilton (1998, S. $247 \mathrm{ff}$ ) zeigen, dass literale Praktiken in unterschiedlichen Bereichen des täglichen Lebens mit Werten, Einstellungen, Gefühlen und sozialen Beziehungen verbunden sind. In Anerkennung dieser sozialen Einbettung von Numeralität betonen

\footnotetext{
1 Finanziert von der Landesforschungsförderung Hamburg (2017-2020); Forschungsverbund aus sechs Teilprojekten und den Partnern Hochschule für Angewandte Wissenschaften (HAW), Universität Hamburg (UHH), Helmut-Schmidt-Universität/Universität der Bundeswehr Hamburg (HSU), UNESCO Institute for Lifelong Learning (UIL).
} 
Evans et al. (2017) im aktuellen Diskurs auch den Aspekt der sog. „,numeralen Umgebung“ (numerate environment).

Viertens ist zu betonen, dass in numeralen Praktiken immer auch das jeweilige gesellschaftliche Fähigkeitsregime zu Kompetenz (Anforderungen und deren Bewältigung) eingelassen ist. Fähigkeit ist zu verstehen als ,,das Vermögen, etwas zu realisieren“ (Weisser 2018, S. 99), während Kompetenz ,aktuale als auch potentielle Fähigkeiten und Fertigkeiten einer Person [erfasst], die jeweils situations-, d.h. anforderungsabhängig sind" (Werner 2017, S. 107) und sich folglich erst in der Performanz realisieren.

Forschungsmethodologisch sind beide Forschungsprojekte bottom-up-orientierten Ansätzen in der empirischen Literalitäts- und Numeralitätsforschung zuzuordnen (Evans et al. 2017). Die Zwischenbefunde in Bezug auf die Kategorien Alter und Behinderung sind für die Grundbildungsforschung auch deshalb von Relevanz, da Ältere und Menschen mit Lernschwierigkeiten im Rahmen der Numeralitätsforschung bisher kaum berücksichtigt wurden (vgl. Tenorth 2004, S. 177).

\subsection{Forschungseinblick 1: Numeralität als soziale Praxis im Wandel der Zeit}

Im Folgenden werden ausgewählte erste Befunde und Annahmen der Studie „Numeralität als soziale Praxis im Wandel der Zeit" vorgestellt. Diese Studie untersucht in einem explorativen Zugang individuelle numerale Praktiken der sog. jungen Alten (60-80 Lebensjahre) und hochbetagter Menschen (ab dem 80. Lebensjahr). Ausgangspunkt sind Befunde aus PIAAC und CiLL, nach denen numerale Kompetenzen bzw. Praktiken in den höheren Alterskohorten gering bis durchschnittlich ausgeprägt sind und im Laufe des weiteren Lebens abnehmen (Knauber und Weiß 2014; Schmidt-Hertha 2018). Ferner verweist eine aktuelle Sekundäranalyse zu CiLL und PIAAC bei älteren Personen u.a. auf geschlechtsstereotype Unterschiede im Umgang mit Finanzangelegenheiten (Redmer und Grotlüschen 2019). Darüber hinaus kann nur wenig über die Anwendung von Alltagsmathematik dieser Alterskohorten ausgesagt werden.

Ziel der Studie ist es, diese Forschungslücke zu schließen, wobei für uns von besonderem Interesse ist, welche Rolle Numeralität im Leben Älterer spielt und mit welchen individuellen Bedeutungszuschreibungen sie verbunden wird. Diesen Zusammenhang haben wir bereits in einer ethnografischen Studie zur Literalität im öffentlichen Raum nachgewiesen (Zeuner und Pabst 2011). Im Vordergrund standen dabei partizipatorische, kulturelle und gesellschaftsverändernde Dimensionen, die sich in individuellen und kollektiven Formen zeigen (Pabst und Zeuner 2016, S. 62). Auch die aktuelle Studie zielt mit der Orientierung an LSP und insbesondere den Studien von Street und Baker (Street 1984; Baker und Street 1996; Street et al. 2008) auf die soziale Einbettung sowie auf den individuellen Umgang und Gebrauch von numeralen Praktiken in unterschiedlichen sozialen Kontexten und in unterschiedlichen biografischen Zusammenhängen. Die Vielfalt des Umgangs mit numeralen Praktiken, ihre Anwendungsmöglichkeiten und Bedeutungszuschreibungen stehen im Vordergrund des Erkenntnisinteresses. Da sich die Studie auf ältere Menschen bezieht, untersuchen wir auf individuell-reflexiver Ebene biographische 
und gesellschaftliche Veränderungen numeraler Praktiken und ihrer Bedeutungen, die sich durch individuelle und gesellschaftliche Entwicklungen ergeben.

Das Erhebungsdesign dieser Studie umfasst zum einen eine punktuelle Untersuchung historischer Quellen und Artefakte zur Numeralität (ab 1920), um Aufschlüsse zum alltäglichen Umgang mit Numeralität aus historischer Perspektive zu gewinnen. Darüber hinaus wurden im Vorfeld 11 Kurzinterviews (Dauer: 5-10 min) mit Menschen im Alter von 15-84 Jahren über ihre Vorstellungen zu Numeralität durchgeführt. Zentral für die Studie sind leitfadengestützte, biografiebezogene Interviews mit älteren Menschen. Ziel der Interviews ist es zu erfahren, wie die Befragten in ihrem bisherigen Leben mit Numeralität umgegangen sind bzw. umgehen und welche persönlichen numeralen Handlungsweisen und Positionen sie entwickelt haben.

Leitfadengestützte Interviews stellen nach Hopf (2015) eine gelungene Methode dar, um einerseits vergleichbare Daten erheben und andererseits differenzierten Perspektiven und individuellen Orientierungen genügend Raum geben zu können. Sie bieten die Möglichkeit eines diskursiven Austausches über den Forschungsgegenstand und unterstützen den Reflexionsprozess bei nicht immer leicht zugänglichen Handlungsaspekten und Wissensformen (ebd., S. $353 \mathrm{ff}$.).

Vor dem Hintergrund des Konzepts NSP wurde ein Leitfaden für vertiefende biographische Interviews entwickelt, in dessen Mittelpunkt die folgenden Aspekte stehen:

- individuelle alltägliche numerale Praktiken und die damit verbundenen Bedeutungszuschreibungen;

- biografische Aspekte zum Umgang mit Numeralität in Bezug auf Kindheit und Jugend, Schulzeit/Ausbildung, Beruf/Erwerbsarbeit, Familie und Ruhestand;

- die individuelle Verortung der befragten Personen im dritten und vierten Lebensalter.

Zum aktuellen Zeitpunkt umfasst unser Sample 17 Interviews mit Personen im Alter von 64 bis 93 Jahren (Dauer: 47 bis $120 \mathrm{~min}$ ). In der Analyse orientieren wir uns am Ansatz der Grounded Theory (Strauss und Corbin 1990), wobei zunächst jegliche numeralen Ereignisse und Praktiken, die in den Interviews zur Sprache kommen, identifiziert und unterschiedlichen Handlungsdomänen und sozialen Kontexten zugeordnet werden. Im weiteren Verlauf stehen die subjektiven Umgangsweisen, Bedeutungszuschreibungen sowie ihre Veränderungen im Rahmen gesellschaftlicher und biografischer Entwicklungen im Vordergrund der Analyse.

Erste Befunde zeigen, dass numerale Praktiken v.a. in den Handlungsdomänen Haushalt, Finanzen, Gesundheit, soziales Leben/Teilhabe angewandt werden. Sie sind in unterschiedlichen Tätigkeiten eingebettet, wie die folgenden Beispiele zeigen:

- Haushalt: Einkaufen, Kochen und Backen, Handarbeit, Gartenarbeit

- Finanzen: (Online-)Banking, Budgets kontrollieren (Wochen-, Monats-/Jahresplanung), Alterssicherung/Rente kontrollieren, Sparen und Vermögensbildung

- Gesundheit: präventive Tätigkeiten (Mentaltraining, Bewegung), Bedienung medizinischer Geräte, Medikation, Arztbesuche 
- Soziale Teilhabe: Pflege von sozialen Netzwerken (Familie, Freunde usw.) Teilnahme an Bildungsveranstaltungen, an kulturellen und politischen Veranstaltungen

Die numeralen Praktiken sind im Rahmen dieser Tätigkeiten individuell verschieden. Während bspw. eine Person beim Backen alle Zutaten präzise abwiegt, orientiert sich eine andere eher an der Konsistenz der Speise, um über die Zugabe von weiteren Zutaten zu entscheiden. Es finden sich zudem Hinweise, dass numerale Praktiken aufgrund routinisierter Handlungen oftmals wenig bewusst ausgeführt werden.

Es finden sich Aussagen über die Verwendung von Haushaltsbüchern oder entsprechender Software, wobei dies nicht immer mit der finanziellen Notwendigkeit einer genauen Budgetplanung verbunden ist. Vielmehr sind diese Praktiken mit Werten wie Sparsamkeit und die Vermeidung von Überflüssigem verbunden, die bereits in der Kindheit erlernt wurden. Dabei werden für sämtliche Ausgaben Kassenbelege gesammelt, z.B. auch für den Coffee-to-Go am Bahnhofskiosk, selbst wenn das Verkaufspersonal über die Bitte um den Beleg irritiert wirkt. Die Buchführung erfolgt zumeist ritualisiert an einem bestimmten Wochentag, am Monatsende oder wenn der Behälter für die Belege voll ist.

Die Befragten berichten über routinisierte numerale Praktiken wie Kopfrechnen oder Überschlagen. Diesen mentalen Handlungen wird besondere Bedeutung beigemessen: Sie sind lebenslang geübte Praxis, die in Kindheit und Schule gelernt und im Erwerbsleben regelmäßig angewandt wurden. Im Alter werden diese numeralen Praktiken in verschiedenen Lebenszusammenhängen bewusst gepflegt, um geistig rege zu bleiben. Gleichzeitig beobachten die Befragten, dass jüngere Generationen in den gleichen Situationen oftmals auf technische Unterstützungen zurückgreifen.

Menschen organisieren im Rahmen ihrer Paarbeziehungen numerale Aufgaben arbeitsteilig. Eine explizit genderstereotype Arbeitsteilung können wir jedoch nicht feststellen. Eine Interviewpartnerin berichtet von ihrer Finanzkalkulation in Eigenregie für den Eigenheimbau, mit der sie ihren Mann von der Realisierbarkeit dieses Vorhabens überzeugte. Auch eine andere Frau berichtet von den Schwierigkeiten, die sie als alleinstehende Frau mit zwei Kindern beim Bau eines Hauses zu bewältigen hatte. Darüber hinaus wird deutlich, dass die arbeitsteilige Organisation bei Paaren im Alter Veränderungen unterliegt, wenn bspw. gesundheitliche Beeinträchtigungen hinzukommen.

Resümierend kann zum jetzigen Zeitpunkt der Untersuchung festgehalten werden, dass je nach Lebenssituation subjektiv differenzierte Gründe dazu führen, weshalb und auf welche Weise bestimmte numerale Praktiken angewandt werden. Ebenso zeigt sich, dass die arbeitsteilige Organisation numeraler Praktiken innerhalb von Paarbeziehungen auf differenzierten und vielfältigen Begründungsmustern beruht. Numeralität stellt ein sehr vielseitiges Konzept dar, bei dem v. a. im Gegensatz zu Literalität der Tatsache sehr viel mehr Beachtung geschenkt werden muss, dass den alltäglichen numeralen Praktiken wesentlich differenziertere mathematische Fähigkeiten zugrunde liegen, als dies bspw. bei der Anwendung von Schriftsprache der Fall ist. Die Aussagen der Interviewten zeigen, welche subjektive Bedeutung unsichtbare numerale Praktiken, wie das Überschlagen von Summen, das (Ab-)Schätzen von Größen oder Gewichten oder die Entwicklung eines Gefühls für Höhen und Ent- 
fernungen usw., haben können. Eine Textgebundenheit (semiotische Kodifizierung) wie bei Literalität ist bei Numeralität nicht immer gegeben.

\subsection{Forschungseinblick 2: Numerale Praktiken von Menschen mit Lernschwierigkeiten}

Die Relevanz von Grundbildung für die Personengruppe von erwachsenen Menschen mit Lernschwierigkeiten manifestiert sich in den Befunden der vorgelagerten Bildungsketten, die deutlich machen, dass das Recht auf Bildung (vgl. UN 2006) für diese Gruppe bislang nur in Ansätzen eingelöst ist (vgl. BMAS 2016, S. 111; KMK 2016, S. XXI) und eine gleichberechtigte Teilhabe an Gesellschaft folglich noch aussteht (BMAS 2016, S. 154). Insgesamt sind die Personen seltener in einer Ausbildung, üben seltener einen Beruf aus und arbeiten vermehrt in einer Werkstatt für behinderte Menschen (vgl. BMAS 2016, S. 119f.; 163; 196). Die wenigen vorliegenden wissenschaftlichen Studien ${ }^{2}$ zu Numeralität bzw. Alltags-/Mathematik folgen darüber hinaus vornehmlich einer defizitorientierten Perspektive (z. B. Peng et al. 2018; siehe Analyse bei Tan et al. 2019). Beeinträchtigungen, Syndromdiagnosen und die geringeren/fehlenden Kompetenzen stehen im Vordergrund und werden als ein rein personengelagertes Merkmal interpretiert (vgl. Tan et al. 2019) und nicht als auch Ausdruck der Zuschreibungen eines Fähigkeitsregimes. Demgegenüber folgt die vorliegende Studie ressourcenorientierten Ansätzen, die die Subjekte in ihrer Handlungsfähigkeit als ,,mathematics doers and thinkers“ (ebd., S. 1) anerkennen.

Ausgangspunkt ist die Frage, wie Menschen mit Lernschwierigkeiten ihren Alltag, in welchem eben auch Rechnen/Mathematik eingelassen sind, bewältigen. In diesem Alltag dokumentiert Behinderung bzw. dis/ability, in Anschluss an Arbeiten der Disability Studies (z. B. Waldschmidt 2019), einen Fähigkeitskonflikt (Weisser 2018): zwischen gesellschaftlichen Erwartungen des Klassifizierungssystems (Kompetenzlevel) einerseits und je subjektiv vorliegenden numeralen Fähigkeiten andererseits. Die Beforschung numeraler Praktiken im Sinne von NSP verspricht, dieses Wechselgefüge aus subjektiver Handlungsfähigkeit, sozialer Einbettung, strukturellen Teilhabemöglichkeiten sowie gesellschaftlichen Klassifizierungssystemen eines Fähigkeitsregimes (Buchner et al. 2015) sichtbar machen zu können. Forschungsleitend ist die Frage, auf welche Weise Menschen mit Lernschwierigkeiten numerale Praktiken im Alltag anwenden, welche numeralen Fähigkeiten sich in diesen Praktiken spiegeln und welche subjektiven Bedeutungen den Praktiken jeweils zugeschrieben werden.

Hierzu kombiniert das qualitative Erhebungsdesign Prinzipien Partizipativer Forschung (von Unger 2014) mit dem methodologischen Ansatz der Grounded Theory (Strauss und Corbin 1990; Strübing 2014) (im Detail: Schreiber-Barsch et al. in Vorbereitung). Den Prinzipien Partizipativer Forschung folgend wurde das Sample in direkter Ansprache generiert, indem das Projekt in einfacher Sprache z. B. an of-

\footnotetext{
2 Im PIAAC-Sample ( $n=5379$ Personen) wurden lediglich 20 Personen (0,4\%) mit Sonder-/Förderschulabschluss berücksichtigt. Personen, die in institutionalisierten Einrichtungen leben, waren im Sampling gänzlich ausgeschlossen (GESIS 2012, S. 7).
} 
fenen Treffpunkten der Personengruppe, bei Selbstvertretungsorganisationen, Lernfesten u. Ä. präsentiert $(n=11)$ und um Mitarbeit durch Menschen mit Lernschwierigkeiten als Expertinnen und Experten des Alltags gebeten wurde. Für alle Samples (s. unten) war leitend, eine Abdeckung von alltäglichen Situationen aus vier Lebenskontexten (Wohnen, Arbeit, Freizeit, berufliche Bildung) zu erreichen. Parallel wurden Forschungsgruppen von Menschen mit Lernschwierigkeiten zusammen mit Studierenden (Universität Hamburg) installiert, um eine kommunikative Validierung der Forschungsschritte und -instrumente sicherzustellen (vgl. Gundlach et al. im Druck).

Das Erhebungsdesign (2018/19) umfasst drei Schritte:

1. Numeralität beobachten: In einem explorativen Zugang wurden sog. Teilnehmende Begleitungen (im Sinne Teilnehmender Beobachtung ${ }^{3}$ ) mit Menschen mit Lernschwierigkeiten durchgeführt. Ziel war, ein Raster zur Differenzierung der Facetten von Numeralität (siehe Tab. 2) zu entwickeln und die auch nicht-bewussten Anwendungsformen (bspw. Routinen) zu erheben ( $n=11$ Begleitungen; Alter: 20 bis 80 Jahre; zwischen 2 bis 7 1/2h) (2018).

2. Numeralität beschreiben: Im Anschluss wurden 13 Interviews mit Menschen mit Lernschwierigkeiten durchgeführt, um subjektive Beschreibungen und Bedeutungszuschreibungen sowie Motive in ihrer sozialen Einbettung zu ermitteln (Alter: 18 bis 42 Jahre; zwischen 30 min bis $2 \frac{1}{2} \mathrm{~h}$ ) (2018).

3. Numeralität als Aufgabe von Professionellen: Im August/September 2019 werden vier Gruppendiskussionen mit professionell Tätigen durchgeführt, um eine kontrastive Spiegelung mit den Begleitungen und Interviews sowie Aussagen über Potentiale und Grenzen der professionellen Unterstützung der Anwendung von Numeralität im Alltag zu erhalten.

Ein erstes Zwischenergebnis ist die Ausdifferenzierung von Facetten von Numeralität (Tab. 2). Die deduktive Synthese gründet auf einschlägigen Einteilungen von Numeralitätskategorien, die für das Sample in der Fähigkeitsauslegung anschlussfähig adaptiert sowie im Zuge des iterativen Auswertungsprozesses der Grounded Theory induktiv erweitert wurden (und derzeit noch werden). Die Kategorien der induktiven Erweiterung können erklären, warum und weshalb auf welche Weise numerale Praktiken (und in ihnen numerale Facetten) angewandt und mit subjektiver Bedeutung belegt werden.

Während die Ergebnisse zu diesen Facetten an anderer Stelle ausführlich dargelegt sind (Schreiber-Barsch et al. in Vorbereitung), sollen hier anhand einer Interviewsequenz die analytischen Bezüge zu numeralen Ereignissen (Events), Praktiken und darin eingelassenen Fähigkeiten im Sinne von Facetten (siehe Tab. 2) exemplarisch verdeutlicht werden.

\footnotetext{
3 Der Begriff „Beobachtung“ konnte nicht verwendet werden, da dieser bei der von immer wiederkehrenden Diagnostiken und Tests sehr häufig belasteten Personengruppe direkt zur Verweigerung bzw. zum Abbruch der Erhebung geführt hätte (so wurden bspw. regelmäßig Rückversicherungen eingeholt, ob es sich tatsächlich nicht um einen „Mathetest“ handeln würde).
} 
Tab. 2 Facetten von Numeralität. (Eigene Darstellung)

\begin{tabular}{|c|c|c|c|}
\hline Facette & Teilkomponente(n) der Facette & & Herleitung \\
\hline $\begin{array}{l}\text { Raum(-ori- } \\
\text { entierung) } \\
\text { und Form }\end{array}$ & Raumerfahrung/-vorstellung & Ortsbestimmung & $\begin{array}{l}\text { Deduktive } \\
\text { Synthese } \\
\text { (in An- }\end{array}$ \\
\hline $\begin{array}{l}\text { Zahlen und } \\
\text { Rechnen }\end{array}$ & $\begin{array}{l}\text { Rechenoperationen/mit Zahl Welt } \\
\text { quantifizieren }\end{array}$ & Zahlensinn/-verständnis & schluss an: \\
\hline $\begin{array}{l}\text { Größen } \\
\text { und Messen }\end{array}$ & $\begin{array}{l}\text { Umgang mit Maßeinheiten } \\
\text { (Umsetzung: angemessene Aus- } \\
\text { wahl von Messgeräten und Maß- } \\
\text { einheiten) (Bsp. Zeit) }\end{array}$ & $\begin{array}{l}\text { Anpassungsleistung } \\
\text { (Anwendung: Messinstru- } \\
\text { ment in Passung mit dem zu } \\
\text { messenden Objekt bringen) }\end{array}$ & $\begin{array}{l}\text { 2017; FHH } \\
\text { 2011; KMK } \\
\text { 2004; ISB } \\
\text { 2003) }\end{array}$ \\
\hline $\begin{array}{l}\text { Muster und } \\
\text { Strukturen }\end{array}$ & Muster und Beziehungen & $\begin{array}{l}\text { Gesetzmäßigkeiten und } \\
\text { Routinen }\end{array}$ & \\
\hline $\begin{array}{l}\text { Wahr- } \\
\text { schein- } \\
\text { lichkeiten, } \\
\text { Häufigkei- } \\
\text { ten, Zufall }\end{array}$ & $\begin{array}{l}\text { Wahrscheinlichkeiten, Unsicher- } \\
\text { heiten erkennen (z.B. Häufigkeits- } \\
\text { verteilungen im Alltag erkennen } \\
\text { und transferieren) }\end{array}$ & - & \\
\hline Artefakte & $\begin{array}{l}\text { Anschauungsmittel (z. B. Bilder, } \\
\text { Listen, Rechnungen, Fahrpläne) }\end{array}$ & $\begin{array}{l}\text { Arbeitsmittel: Technologien, } \\
\text { Gegenstände, Objekte (z. B. } \\
\text { örtliche Orientierungspunkte), } \\
\text { menschliche Funktionsträger } \\
\text { (z.B. Assistenz) }\end{array}$ & \\
\hline $\begin{array}{l}\text { Soziale } \\
\text { Einbettung }\end{array}$ & $\begin{array}{l}\text { Gesellschaftlicher Kontext (z.B. } \\
\text { Bedeutung von Pünktlichkeit) }\end{array}$ & $\begin{array}{l}\text { Institutioneller Kontext (z. B. } \\
\text { vorgegebene Zeitstrukturen } \\
\text { am Arbeitsplatz) }\end{array}$ & $\begin{array}{l}\text { Induktive } \\
\text { Erweiterung } \\
\text { (im Aufbau) }\end{array}$ \\
\hline $\begin{array}{l}\text { Subjektive } \\
\text { Bedeutsam- } \\
\text { keit }\end{array}$ & $\begin{array}{l}\text { Zuschreibung subjektiver Bedeut- } \\
\text { samkeit generiert Lernanlässe für } \\
\text { Numeralität (z. B. Wegstrecke zum } \\
\text { Hobby überwinden; Geburtstags- } \\
\text { daten nur von Freunden lernen) }\end{array}$ & - & \\
\hline $\begin{array}{l}\text { Direkte In- } \\
\text { teraktionen }\end{array}$ & $\begin{array}{l}\text { Direkte (verbal und nonverbal) } \\
\text { soziale Interaktion und Kommuni- } \\
\text { kation mit bestimmten Personen }\end{array}$ & - & \\
\hline
\end{tabular}

\subsubsection{Interviewsequenz: Eckenrechnen (I_3(\#84-88\#))}

[...] Also beim Rechnen war ich FRÜHER in der Schule eher eine Niete. In der Berufsschule war das dann ganz lustig, da war ich dann immer der Schnellste ... komischerweise. Wir haben MORGENS immer zum Unterrichtsbeginn Eckenrechnen gemacht. Das heißt, alle haben sich in eine Ecke gestellt. Und wenn man FERTIG war, dann ... Also wenn man die Aufgabe ... Hat der Lehrer eine Aufgabe gestellt und wenn man die als SCHNELLSTES konnte, konnte man immer eine Ecke weiter. So. Ganz am Anfang war ich immer der Letzte. Und zum Ende hin des Schuljahres war ich der Schnellste. Und dann haben die Mitschüler gefragt: Wie kannst du das? Wie kriegst du so schnell dieses Ergebnis raus? Ja, ich hab gesagt: Ich spiele Computer. Haben die gesagt: Du verarschst uns. Nein, ich hab es gibt ein SPIEL, das heißt (OMSI), ein BusSimulator. Und der ist so realistisch, dass man sogar FAHRKARTEN verkau- 
fen kann. Und der zeigt dann nicht an, wie viel Geld man noch GEBEN muss, sondern man muss das rechnen. So, man hat maximal $10 \mathrm{~s}$ Zeit oder $5 \mathrm{~s}$ Zeit, dann fangen die Fahrgäste an zu meckern: Wo ist mein Rückgeld? Du hast mir zu VIEL Rückgeld gegeben. Du hast mir zu WENIG Rückgeld gegeben. Das heißt, das muss man alles ausrechnen. Ja. (I_3 = Interview 3; Großschreibung in der Transkription markiert eine Betonung)

Die Passage illustriert die Verflechtung von Lebenskontexten und Lernformen in der Ausübung numeraler Praktiken. In den Ereignissen zeigt sich als Anlass zur Ausführung numeraler Aktivitäten sowohl die schulische Situation des Mathematikunterrichts, die berufsschulische Situation des Eckenrechnens als auch das Computerspiel (Artefakt) in der Freizeit. Die Bewältigung der numeralen Anforderung (Kopfrechnen unter Zeitdruck) lernt die Person schließlich nicht-intentional über das Verkaufen von Fahrkarten beim Computerspiel und kann dieses implizit erworbene Können auf das „Eckenrechnen“ in der Berufsschule übertragen. Es spiegeln sich zudem Fähigkeiten in der erfolgreichen Anwendung der numeralen Facetten Größen und Messen (u. a. Zeit als Zeitdruck; als Orientierung in der Zeit, z. B. ,früher“, ,als SCHNELLSTES“), Zahlen und Rechnen (Rechnen, Umgang mit Geld, Mengen (i. S. des Zählens)) sowie Muster und Strukturen (Zusammenhang zwischen Zeit und zu bewältigender Aufgabe).

Die Analyseperspektive von NSP öffnet den Blick darauf, dass die Person Numeralität nicht nur in vielfältiger Weise, sondern subjektiv bedeutsam und sozial eingebettet verwendet und anpasst. Die soziale Einbettung zeigt sich z. B. in der Reproduktion von Kompetenzklassifizierungen (besser-schlechter), sowohl bei der Lehrkraft (in der kompetitiven Leistungsabfrage Eckenrechnen) als auch bei der Person (,eine Niete“ vs. ,immer der Schnellste“). Schließlich initiiert das persönlich bedeutsame, da auf eigenen Interessen beruhende Computerspielen eine lernende Erweiterung des numeralen Könnens der Person - das auf die formale Bildungssituation rückwirkt. Erst das Zusammenspiel kann mithin die Komplexität und Begründungsstrukturen numeraler Praktiken erklären.

\section{Plädoyer für eine subjektorientierte Grundbildung}

Auf der Grundlage der Auswertung des Forschungsstands und der ersten Befunde unserer Forschungsprojekte möchten wir für eine Perspektiverweiterung des Grundbildungsdiskurses durch den Ansatz einer subjektorientierten Grundbildung plädieren. Für Grundbildung bleibt die Debatte um spezifische Inhalte, Kompetenzen und Lernziele wesentlich, um die gesellschaftlich fundierte und legitimierte Absicherung einer pädagogischen Vermittlung von teilhabeeröffnenden Grundfertigkeiten zum Ziele einer möglichst autonomen (d.h. selbstbestimmten, nicht notwendigerweise unabhängigen) Lebensführung Erwachsener sicherzustellen. Dies bleibt allerdings ohne eine subjektorientierte Erweiterung der Perspektive, die Lernumgebungen, Interessen, je eigene Gründe für Lernanlässe, Dilemmata und Widersprüche mitdenkt, unvollständig. 
Unsere Forschungen unterstreichen die Annahme, dass das numerale Handeln der befragten bzw. begleiteten Personen nicht defizit-, sondern ressourcenorientiert erhoben werden sollte, da sie unabhängig von eindeutig benennbaren und messbaren Kompetenzen vielfältige numerale Praktiken in ihrem Alltags- und Arbeitsleben und darüber hinaus ausüben und damit erkennbar ziel- und ergebnisorientiert handeln; bedeutsam ist dieser Kontext für Möglichkeiten der Partizipation. Damit grenzen sich unsere Projekte von defizitorientierten Untersuchungen über (alltags-)mathematische Fähigkeiten und Fertigkeiten Erwachsener ab, die darauf abzielen, diese zu systematisieren, zu messen, zu klassifizieren und damit einem außen- und interessengeleiteten Bewertungssystem zu unterwerfen. Eine solche Vorgehensweise beruht auf bestimmten gesellschaftlichen Vorannahmen und Erwartungshaltungen bezogen auf Numeralität als notwendiger Kulturtechnik auf der einen und Zuschreibungen an eine bestimmte Performanz auf der anderen Seite, vernachlässigen hingegen den Blick auf die subjektiv bedeutsame Ausübung numeraler Praktiken, und die hierin eingelassenen (subjektgebundenen) Fähigkeiten und Fertigkeiten.

Unsere Überlegungen und vorläufigen Ergebnisse zeigen, dass Menschen, unabhängig von ihrem formalen mathematischen Wissen, im Alltagshandeln numerale Praktiken in ihrem eigenen Sinne anwenden und diese auch weiterentwickeln, verwerfen, verändern und damit nicht nur unmittelbare Anforderungen oder fremde Interessen bedienen, sondern sie auch im Sinne vorausschauenden Handelns anwenden.

Sie geben zudem Hinweise, auf welche Weise die vielfältigen numeralen Praktiken, die Menschen anwenden, auch für die didaktische Gestaltung in der Grundbildung für Erwachsene fruchtbar gemacht werden können. Die meisten der bisher entwickelten Curricula basieren auf defizitorientierten Perspektiven und versuchen, mathematische Grundlagen und Rechenverfahren zu vermitteln (Kittel 2016, S. 431). Selbst der dritte Teil des DVV-Curriculums (DVV 2017), der sich explizit auf Alltagssituationen Erwachsener bezieht, zielt letztlich v.a. auf die Vermittlung von Rechenkenntnissen bezogen auf bestimmte Anwendungsgebiete. Damit werden sie zwar kontextualisiert und Lernende können sie u. U. auch leichter nachvollziehen und einordnen, aber das grundlegende Problem einer Defizitorientierung bleibt bestehen. Wir sprechen uns hingegen dafür aus, ausgehend von vorhandenen Kenntnissen und Anwendungserfahrungen, Lernwege und -prozesse gemeinsam mit den Lernenden zu entwickeln. Dies setzt voraus, dass sich Lehrende auf die Perspektive von Lernenden einlassen, bestimmte vorgeformte Erwartungen und Einschätzungen über Fähigkeiten und Kompetenzen zurückstellen und die numeralen Praktiken der Lernenden ernstnehmen, wertschätzen, als subjektiv zielführende Anwendungen anerkennen und davon ausgehend mit den Lernenden weiterarbeiten.

Open Access Dieser Artikel wird unter der Creative Commons Namensnennung 4.0 International Lizenz (http://creativecommons.org/licenses/by/4.0/deed.de) veröffentlicht, welche die Nutzung, Vervielfältigung, Bearbeitung, Verbreitung und Wiedergabe in jeglichem Medium und Format erlaubt, sofern Sie den/die ursprünglichen Autor(en) und die Quelle ordnungsgemäß nennen, einen Link zur Creative Commons Lizenz beifügen und angeben, ob Änderungen vorgenommen wurden. 


\section{Literatur}

Baker, D., \& Street, B. (1996). Literacy and numeracy models. In A. Tuijnam (Hrsg.), International encyclopedia of adult education and training (2. Aufl. S. 79-85). Oxford: Pergamon Press.

Barton, D., \& Hamilton, M. (1998). Local literacies: reading and writing in one community. London: Routledge.

BMAS - Bundesministerium für Arbeit und Soziales (2016). Zweiter Teilhabebericht der Bundesregierung über die Lebenslagen von Menschen mit Beeinträchtigungen. Teilhabe-Beeinträchtigung-Behinderung. https://www.bmas.de/SharedDocs/Downloads/DE/PDF-Publikationen/a125-16teilhabebericht.pdf;jsessionid=29CAB83561B784C4FBAA1F908D788760?_blob=publicationFile \&v=9. Zugegriffen: 1. Juli 2019.

Buchner, T., Pfahl, L., \& Traue, B. (2015). Zur Kritik der Fähigkeiten: Ableism als neue Forschungsperspektive der Disability Studies und ihrer Partner_innen. Zeitschrift für Inklusion, 2, https://www. inklusion-online.net/index.php/inklusion-online/article/view/273. Zugriffen: 1. Juli 2019.

Coben, D., Colwell, D., Macrae, S., Boaler, J., Brown, M., \& Rhodes, V. (2003). Adult numeracy: review of research and related literature. http://dera.ioe.ac.uk/22487/1/doc_2802.pdf. Zugegriffen: 1. Juli 2019.

Cockcroft, W. (1982). Mathematics counts. London: HMSO.

D'Ambrosio, U. (1985). Ethnomathematics and its place in the history and pedagogy of mathematics. For the Learning of Mathematics, 5(1), 44-47.

DVV - Deutscher Volkshochschulverband (2017). Rechnen. DVV-Rahmencurriculum (2. Aufl.), Bonn. https://www.volkshochschule.de/microsite/grundbildung/unterrichten/Curricula.php. Zugegriffen: 15. Mai 2019.

Evans, J., Yasukawa, K., Mallows, D., \& Creese, B. (2017). Numeracy skills and the numerate environment: affordances, opportunities, supports and demands. Adults Learning Mathematics, 12(1), 17-26.

FHH - Freie und Hansestadt Hamburg, Behörde für Schule und Berufsbildung (2011). Bildungsplan Grundschule. Mathematik. Hamburg. https://www.hamburg.de/contentblob/2481796/e71dafe076bf5 97d320c6a76ae57263c/data/mathematik-gs.pdf. Zugegriffen: 1. Juli 2019.

Friebe, J., Schmidt-Hertha, B., \& Tippelt, R. (Hrsg.). (2014). Kompetenzen im höheren Lebensalter. Ergebnisse der Studie „Competencies in Later Life“ (CiLL). Bielefeld: wbv.

Geiger, V., Goose, M., \& Forgasz, H. (2015). A rich interpretation of numeracy for the 21st century: a survey of the state of the field. ZDM - The International Journal on Mathematics Education, 47(4), 531-548. https://doi.org/10.1007/s11858-015-0708-1.

GESIS (2012). PIAAC Deutschland 2012 - Hintergrundfragebogen. https://www.gesis.org/fileadmin/ piaac/Downloadbereich/PIAAC_BQ_Final_deutsch.pdf. Zugegriffen: 1. Juli 2019.

Grotlüschen, A., Buddeberg, K., Dutz, G., Heilmann, L., \& Stammer, C. (2019). LEO. Leben mit geringer Literalität. Hamburg: Pressebroschüre.

Gundlach, H., Panzer, M., Rückforth, B., \& Schreiber-Barsch, S. (i.E.). Lebenslanges Lernen ${ }^{\text {inklusiv. Ein- }}$ blicke in das Lehrprojekt „Partizipative Forschungswerkstatt“ an der Universität Hamburg. Erwachsenenbildung und Behinderung, 2, 49-61.

Heath, S. B. (1982). What no bedtime story means: narrative skills at home and at school. Language in Society, 11(1), 49-76.

Hopf, C. (2015). Qualitative Interviews - ein Überblick. In U. Flick, E. von Kardorff \& I. Steinke (Hrsg.), Qualitative Forschung: Ein Handbuch (S. 349-360). Reinbek bei Hamburg: Rowohlt.

ISB - Staatsinstitut für Schulqualität und Bildungsforschung München (2003). Lehrpläne Förderschwerpunkt Geistige Entwicklung-Grund- und Hauptschulstufe. Mathematik. https://www.isb.bayern.de/ download/9014/mathematik.pdf. Zugegriffen: 1. Juli 2019.

Kaiser, H. (2009). Bausteine für ein Konzept zur Förderung alltagsmathematischer Kompetenz. Zürich: Schweizerischer Verband für Weiterbildung.

Kittel, A. (2016). Mathematische Grundbildung im Erwachsenenalter. In C. Löffler \& J. Korfkamp (Hrsg.), Handbuch zur Alphabetisierung und Grundbildung Erwachsener (S. 422-435). Münster: Waxmann.

KMK - Konferenz der Kultusminister der Länder in der Bundesrepublik Deutschland (2004). Bildungsstandards im Fach Mathematik für den Primarbereich. Beschluss vom 15.10.2004. Luchterhand. https://www.kmk.org/fileadmin/veroeffentlichungen_beschluesse/2004/2004_10_15Bildungsstandards-Mathe-Primar.pdf. Zugegriffen: 1. Juli 2019.

KMK - Konferenz der Kultusminister der Länder in der Bundesrepublik Deutschland (2016). Sonderpädagogische Förderung in Schulen 2005-2014. Statistische Veröffentlichung der Kultusministerkonferenz. Dokumentation Nr. 210. https://www.kmk.org/fileadmin/Dateien/pdf/Statistik/ Dokumentationen/Dok_210_SoPae_2014.pdf. Zugegriffen: 1. Juli 2019. 
Knauber, C., \& Weiß, C. (2014). Alltagsmathematische Kompetenz. In J. Friebe, B. Schmidt-Hertha \& R. Tippelt (Hrsg.), Kompetenzen im höheren Lebensalter. Ergebnisse der Studie „Competencies in Later Life“ (CiLL) (S. 81-95). Bielefeld: W. Bertelsmann.

Lave, J. (1988). Cognition in practice. Mind, mathematics and culture in everyday life. Cambridge: Cambridge University Press.

Mania, E., \& Tröster, M. (2014). Finanzielle Grundbildung - ein Kompetenzmodell entsteht. Hessische Blätter für Volksbildung, 64(2), 136-145.

Mania, E., \& Tröster, M. (2015). Finanzielle Grundbildung. Programme und Angebote planen. Bielefeld: wbv. https://doi.org/10.3278/43/0049w.

Meyerhöfer, W. (2017). Einführung. In DVV Deutscher Volkshochschulverband (Hrsg.), Rechnen. DVVRahmencurriculum (2. Aufl. S. 3-5). Bonn: DVV.

Pabst, A., \& Zeuner, C. (2016). Lesen und Schreiben. Kulturtechnik oder soziale Praxis? In C. Löffler \& J. Korfkamp (Hrsg.), Handbuch zur Alphabetisierung und Grundbildung Erwachsener (S. 59-72). Münster: Waxmann.

Peng, P., Wang, C., \& Namkung, J. (2018). Understanding the cognition related to mathematics difficulties: a meta-analysis on the cognitive deficit profiles and the bottleneck theory. Review of Educational Research, 88(3), 434-476.

Rammstedt, B. (Hrsg.) (2013). Grundlegende Kompetenzen Erwachsener im internationalen Vergleich: Ergebnisse von PIAAC 2012. Münster: Waxmann.

Redmer, A., \& Grotlüschen, A. (2019). Alltagsmathematische Praktiken im höheren Lebensalter. Zeitschrift für Weiterbildungsforschung. https://doi.org/10.1007/s40955-019-0135-y.

Schmidt-Hertha, B. (2018). Kompetenzerwerb im Lebenslauf - Ergebnisse aus PIAAC und CiLL. In C. Hof \& H. Rosenberg (Hrsg.), Lernen im Lebenslauf: Theoretische Perspektiven und empirische Zugänge (S. 121-139). Wiesbaden: Springer.

Schreiber-Barsch, S., Curdt, W., \& Gundlach, H. (i. V.). Whose voices matter? Adults with learning difficulties and the emancipatory potential of numeracy practices. ZDM-International Journal on Mathematics Education, vorauss. 2, 2020.

Strauss, A., \& Corbin, J. (1990). Basics of qualitative research. Grounded theory procedures and techniques. California: SAGE.

Street, B. (1984). Literacy in theory and practice. Cambridge: Cambridge University Press.

Street, B., Baker, D., \& Tomlin, A. (2008). Navigating numeracies. Home/school numeracy practices. Dordrecht: Springer.

Strübing, J. (2014). Grounded Theory: Zur sozialtheoretischen und epistemologischen Fundierung eines pragmatistischen Forschungsstils. Wiesbaden: Springer.

Tan, P., Lambert, R., Padilla, A., \& Wieman, R. (2019). A disability studies in mathematics education review of intellectual disabilities: directions for future inquiry and practice. Journal of Mathematical Behavior. https://doi.org/10.1016/j.jmathb.2018.09.001.

Tenorth, H. E. (2004). Stichwort: „Grundbildung“ und „Basiskompetenzen“. Herkunft, Bedeutung und Probleme im Kontext allgemeiner Bildung. Zeitschrift für Erziehungswissenschaft, 7(2), 169-182.

The New London Group (1996). A pedagogy of multiliteracies. Designing social futures. Havard Educational Review, 66(1), 60-92.

von Unger, H. (2014). Partizipative Forschung. Einführung in die Forschungspraxis. Wiesbaden: Springer.

UN - United Nations (2006). Convention on the rights of persons with disabilities. http://www.un.org/ disabilities/documents/convention/convention_accessible_pdf.pdf. Zugegriffen: 1. Juli 2019.

Waldschmidt, A. (2019). Handbuch Disability Studies. Wiesbaden: Springer.

Weisser, J. (2018). Inklusion, Fähigkeiten und Disability Studies. In T. Sturm \& M. Wagner-Willi (Hrsg.), Handbuch schulische Inklusion (S. 93-106). Opladen, Toronto: Barbara Budrich.

Werner, B. (2017). Teilhabe durch Grundbildung. Die Förderung Benachteiligter im Sekundarbereich I. Stuttgart: Kohlhammer.

Wygotsky, L. (1986). Thought and language. Cambridge: MIT Press.

Yasukawa, K., Jackson, K., Kane, P., \& Coben, D. (2018a). Mapping the terrain of social practice perspectives of numeracy. In K. Yasukawa, A. Rogers, K. Jackson \& B. Street (Hrsg.), Numeracy as social practice. Global and local perspectives (S. 3-17). London, New York: Routledge.

Yasukawa, K., Rogers, A., Jackson, K., \& Street, B. V. (Hrsg.). (2018b). Numeracy as social practice. Global and local perspectives. London, New York: Routledge.

Zabal, A., Martin, S., Klaukien, A., Rammstedt, B., Baumert, J., \& Klieme, E. (2013). Grundlegende Kompetenzen der erwachsenen Bevölkerung in Deutschland im internationalen Vergleich. In B. Rammstedt (Hrsg.), Grundlegende Kompetenzen Erwachsener im internationalen Vergleich: Ergebnisse von PIAAC 2012 (S. 31-76). Münster: Waxmann. 
Zeuner, C., \& Pabst, A. (2011). „Lesen und Schreiben eröffnen eine neue Welt!“ Literalität als soziale Praxis - eine ethnographische Studie. Bielefeld: W. Bertelsmann.

Publisher's Note Springer Nature remains neutral with regard to jurisdictional claims in published maps and institutional affiliations. 